related with the introduction of biologic Disease-Modifying Antirheumatic Drugs (bDMARDs) and the uninterrupted monitoring following the transition of young patients to adult rheumatology settings.

Objectives: To capture the contemporary ERA profile in Northern Greek patients by analyzing the characteristics and treatment outcome in the era of bDMARDs. Methods: This retrospective cohort study included patients who had been monitored on a 3-month schedule for $\geq 12$ months, from 2000 to 2017. The periodic metric assessment included the disease status and burden by applying contemporary tools in respect to activity, clinical remission (CR) and damage (cJADAS, JSpADA, Wallace criteria for CR and JADI, respectively).

Results: Forty-three patients, mainly male (60\%) with a mean age at disease onset of 10.75 (SD:2.75) years were enrolled. The predominant joints were the hip, ankle and sacroiliac (56\%, $49 \%$ and $46 \%$, respectively). Median lag time from diagnosis to bDMARDs initiation was 8.5 months. Patients with sacroiliitis were more likely to receive bDMARDs (hazard ratio [HR]:3.26, 95\% confidence interval $[\mathrm{Cl}]: 1.35,7.88)$. Thirty six patients $(84 \%)$ achieved clinical remission (CR) on medication (CRONM), within a median time of 11 months and correlated with compliance (HR:3.62, 95\% Cl: 1.34, 9.76). Twenty patients (47\%) experienced a flare following $\mathrm{CR}$, mainly as a single episode (75\%). The median flare-free survival following remission on and off medication (CROFFM) was 42 and 34 months, respectively. At the last evaluation, both median baseline cJADAS (8), and JSpADA (2) dropped to 0, while 13 patients (30\%) were in CROFFM, 17 $(40 \%)$ in CRONM, and $13(30 \%)$ had persistent disease activity. The median percentage of CR per patient was $54 \%$ and no patient had JADI $>0$

Conclusion: Early administration of bDMARDs and compliance to monitoring and treatment improved the long-term outcome in ERA. Axial involvement emerged as a negative prognostic factor with an increased need for bDMARDs and diminished rates of $C R$.

Disclosure of Interests: Dimitrios Deligeorgakis: None declared, Maria Trachana: None declared, Polyxeni Pratsidou-Gertsi: None declared, Despoina Dimopoulou: None declared, Anna Bettina Haidich: None declared, Alexandros Garyfallos Grant/research support from: MSD, Aenorasis SA, Speakers bureau: MSD, Novartis, gsk

DOI: 10.1136/annrheumdis-2020-eular.2946

\section{AB0977 DISEASE COURSE AND TREATMENT RESPONSES IN JUVENILE SYSTEMIC LUPUS ERYTHEMATOSUS; A SINGLE CENTER EXPERIENCE}

B. Sözeri ${ }^{1}$, F. Demir ${ }^{1}$, D. Kilit ${ }^{2}$, C. Pehlivanoğlu ${ }^{3} .{ }^{1}$ University of Health Sciences, Umraniye Training and Research Hospital, Pediatric Rheumatology, Istanbul, Turkey: ${ }^{2}$ Umraniye Traning and Research Hospital, Pediatrics, Istanbul, Turkey; ${ }^{3}$ University of Health Sciences, Umraniye Training and Research Hospital, Pediatric Nephrology, Istanbul, Turkey

Background: Systemic lupus erythematosus (SLE) is a chronic autoimmune inflammatory disease that may cause morbidity and mortality by affecting multiple systems. The $10-20 \%$ of patients have juvenile onset and this cluster have may more severe kidney, neuropsychiatric or hematological involvement.

Objectives: The aim of this study was to assess the clinical and laboratory characteristics, disease activity, and treatment response of patients with juvenile SLE (jSLE).

Methods: This is a retrospective study involving patients between 1 July 2016 and 1 January 2020. The data of patients diagnosed with jSLE and followed up for a minimum of 6 months, were collected. The SLEDAI-2K scores at initiation and at the follow-up (1st, 3rd, 6th, and 12th months of treatment) were examined. The SLEDAI-2K score was considered to be $\leq 4$, for disease remission status. Results: A total of 49 children were included in to the study. The female/male ratio was $4.4 / 1$ and the median age of the patients at the diagnosis was 13 (IQR: 11.115.2) years. The median follow-up of patients was 19 (IQR: 12-25) month. Four of the patients were diagnosed with monogenic SLE. Two siblings were diagnosed with $\mathrm{c} 3$ deficiency and two were diagnosed with familial chilblain lupus. The most common clinical findings were found musculoskeletal complaints $(69.4 \%)$, malar rash $(51 \%)$, oral ulcers (38.8\%), and fever (30.6\%), respectively in over all the group. The frequency of involvement of the system and organs was as follows; mucocutaneous $77.6 \%$, musculoskeletal $69.4 \%$, renal $44.9 \%$, hematological $34.7 \%$, serous membranes $16.3 \%$, neuropsychiatric $12.2 \%$, respectively. All patients had anti-nuclear antibody positivity, while $46.9 \%$ had anti-ds DNA, $14.3 \%$ had anti-Sm and $8.2 \%$ had antiphospholipid antibody positivity. While all patients received hydroxychloroquine treatment, $22.4 \%$ of the patients were received were mycophenolate mofetil, $22.4 \%$ were azathioprine, $14.3 \%$ cyclophosphamide, $12.2 \%$ methotrexate and $10.2 \%$ were rituximab. The median SLEDAI-2K score was 14 (IQR: 10-18.5) at admission, besides it was found to 6 (IQR: 4-12), 4 (IQR: 2-6), 2 (IQR: 0-6) in the 1 st, 6th and 12th months of treatment, respectively. While $98 \%$ of the patients had active disease at admission, $67.3 \%$ at 1 months, $32.7 \%$ at 6 months and $22.4 \%$ at 12 months still had active disease (SLEDAI-2K $>4$ ). Patients with initially high SLEDAI-2K scores had significantly lower remission rates in the first month $(p=0.003)$. It was observed that patients with high SLEDAI-2K scores in admission were more resistant to conventional immunosuppressive treatments and the use of rituximab was more frequent in these patients. At least one major organ (renal, hematological, neurological) were affected in $57 \%$ of patients. The remission rate of these patients at 6 months was found significantly decreased compared to the others $(p<0.005)$ Renal biopsy was performed in 21 patients (42.9\%). 12 of them had type 4 lupus nephritis (LN), 5 had type 2, 2 had type 3, and 1 had type 5. It was observed that patients with renal involvement were the group that reached remission latest.

Conclusion: The presence of high initial SLEDAI-2K scores and the major organ involvement have poor predictive value to achieve inactive disease.

References:

[1] Yee CS, Farewell VT, Isenberg DA, Griffiths B, Teh LS, Bruce IN, et al. The use of Systemic Lupus Erythematosus Disease Activity Index-2000 to define active disease and minimal clinically meaningful change based on data from a large cohort of systemic lupus erythematosus patients. Rheumatology (Oxford) 2011:50:982-8

[2] Romo-Tena J, la Garza RR, Bartnicki-Navarrete I, Alcocer-Varela J, Gómez-Martin D. Factors associated with remission in patients with systemic lupus erythematosus: new insights into a desirable state. Clin Rheumatol 2018;37:3033-3042.

Disclosure of Interests: None declared

DOI: 10.1136/annrheumdis-2020-eular.6305

\section{AB0978 \\ EFFICACY OF ANAKINRA TREATMENT IN PEDIATRIC RHEUMATIC DISEASES; A SINGLE-CENTER} EXPERIENCE

F. Demir ${ }^{1}$, E. Gürler ${ }^{2}$, B. Çolak ${ }^{2}$, B. Sözeri ${ }^{1} .{ }^{1}$ University of Health Sciences, Umraniye Training and Research Hospital, Pediatric Rheumatology, Istanbul, Turkey; ${ }^{2}$ Umraniye Traning and Research Hospital, Pediatrics, Istanbul, Turkey

Background: Anakinra, a recombinant IL-1 receptorantagonist, is a treatment option that acts byblocking the biological activity of $\mathrm{IL}-1$ in autoinflammatory conditions. The diseases that the IL-1 was over expressed are the potentia conditions for this treatment. Such as familial Mediterranean fever (FMF), cryopyrin-associated periodic syndrome (CAPS), and hyperimmunglobulin D syndrome (HIDS) with monogenic inheritance, and systemic juvenile idiopathic arthritis (SoJIA) or idiopathic recurrent pericarditis as non-Mendelian polygenic diseases can be listed as examples of these diseases.

Objectives: The aim of this study was to review the efficacy of anakinra treatment in children with rheumatic disease followed in our center.

Methods: The study group consisted of children with pediatric rheumatic diseases followed up in the Pediatric Rheumatology Department of University of Health Sciences and treated with anakinra (anti-IL 1) for at least one month, between 1 July 2016 and 1 January 2020. The data of these patients were collected retrospectively. The disease activity of the patients at 3rd month and 12th month after the treatment were assessed. We aim to report our experiences of pediatric rheumatic diseases treated with anakinra.

Results: There were 28 patients treated with anakinra for the different pediatric rheumatic diseases. The diagnoses of these patients were as follows; eight were macrophage activation syndrome (MAS) complicating SoJIA, six were HIDS, four were CAPS, four were FMF, four were idiopathic recurrent pericarditis, one was deficiency of interleukin-36 receptor antagonist (DITRA) and one was undefined systemic autoinflammatory disease. $46.4 \%$ of the patients were male and $53.6 \%$ were female. The median age of diagnosis of the patients was 6.5 ((interquartile range (IQR): 4-12.7) years. The median follow-up duration of the patients was 14 (IQR: 3.7-28) months. The patients median anakinra treatment duration was 3 (IQR: 1-4) months. Fever reduced and C-reactive protein normalized within median 2 (IQR: 1-3) and 5 (IQR: 5-7) days, respectively. In the 3rd month after treatment; It was observed that $53.6 \%$ of patients achieved a complete remission (no attack was seen or MAS was improved). The frequency of attacks were decreased more than $50 \%$ in $35.7 \%$ of patients and less than $50 \%$ in $7.1 \%$. $3.6 \%$ of patients were unresponsive to treatment. In the 12th month assessment after the initiation of treatment, it was observed that $28.6 \%$ of patients were still under anakinra treatment and in remission, $10.7 \%$ of them were in remission without anakinra treatment. In $60.7 \%$ of patients, anakinra was switch to other biological treatments for different reasons $(35.7 \%$ partial response or unresponsiveness $17.8 \%$ injection site reactions and $7.1 \%$ daily-injection difficulty). Biologic drug switch to canakinumab and tocilizumab was observed in $88.2 \%$ and $11.8 \%$ of patients, respectively. One patient developed recurrent MAS episodes when the anakinra dose was tapered, and one another patient was unresponsive to the anakinra and dead due to secondary to MAS.

Conclusion: Anakinra seems to be a successful treatment to achieve inactive disease in a significant portion of patients in the early period. The recurrence of disease attacks while drug tapering and injection site reactions were appears the main causes of treatment switch or discontinuation. 